\title{
EFFECT OF AMINO ACID SUBSTITUTION IN NEW DELHI METALLO- $\beta$-LACTAMASE ON CARBAPENEM SUSCEPTIBILITY
}

\author{
AZER ÖZAD DÜZGÜN* \\ Faculty of Engineering and Natural Sciences, Department of Genetics and Bioengineering, \\ Gümüşhane University, Gümüşhane, Turkey
}

(Received: 2 January 2018; accepted: 1 March 2018)

\begin{abstract}
The aim of this work was investigation of clinically important amino acid substitutions of NDM-1 variants. A bla $a_{\mathrm{NDM}-1}$ gene was cloned into expression vector $\mathrm{pET} 100 / \mathrm{D}-\mathrm{TOPO}$. The sequence of NDM-1 variants with substituted amino acids was determined by ClustalW program. A pET100/D-TOPO + bla $a_{\mathrm{NDM}-1}$ was used to generate the alanine mutations at different positions, such as NDM-2 (P28A), NDM-3 (D95A), NDM-4 (M154A), NDM-5 (V88A), NDM-7 (D130A), and NDM-9 (E152A). The mutant variants were transformed into Escherichia coli DH5 $\alpha$. Changes in the activities of alanine mutation variants were determined by E-test. All samples had $32 \mu \mathrm{g} / \mathrm{ml}$ MIC values against ampicillin. The $28^{\text {th }}$ amino acid mutation sample had the highest MIC value against ceftazidime, whereas decreased MIC value for piperacillin. It was observed that the resistance to imipenem was increased in mutant variants D95A, M154A, D130A, and E152A, comparing with P28A and V88A. It was found that NDM-1 has $0.64 \mu \mathrm{g} / \mathrm{ml}$ and the $130^{\text {th }}$ amino acid mutation sample has $0.75 \mu \mathrm{g} / \mathrm{ml}$ meropenem MIC value.
\end{abstract}

Keywords: MBL, mutation, NDM-1, E-test

\section{Introduction}

New Delhi metallo- $\beta$-lactamase-1 (NDM-1) is the most recently discovered Ambler Class B $\beta$-lactamase enzyme. NDM-1 was first isolated from an Indian patient living in Sweden in 2008 and it was described in Klebsiella pneumoniae and Escherichia coli strains [1,2]. The phenotypic tests performed showed that both isolates carry carbapenem resistance due to the production of metallo- $\beta$-lactamase (MBL), but the polymerase chain reaction (PCR) analysis did not detect known MBL genes. Results of the cloning and sequencing studies have shown that MBL was a new enzyme [1,3] and it was called NDM-1 [3].

*E-mails: azerozad@windowslive.com; azer@gumushane.edu.tr 
NDM-2 variant was identified in 2011 and a single amino acid substitution was found to be different from NDM-1 [4]. Altogether, 16 variants of NDM-1 with the amino acid changes are recorded in the literature from 2009 to 2015 (http://www.lahey.org/Studies/other.asp). As with other class B $\beta$-lactamase, NDM-1 also contains zinc ion at the active site [5-7]. NDM-1 is able to hydrolyze all $\beta$-lactams except aztreonam, which is a monobactam [6]. Most of the NDM-1-positive bacteria show resistance to $\beta$-lactams, also other drug classes, and carry resistance mechanisms, such as aminoglycosides and fluoroquinolones [8-12].

After the discovery of NDM-1, it was observed throughout the world in many species of bacteria. NDM-1 has been found in Germany, India, England, Canada, America, Kenya, Israel, South Africa, South Korea, Thailand, many European countries, and Far East countries, such as China [8, 13-15] so far. NDM-1 has also been first described in Turkey in 2011 [16].

In the study of Iraz et al. [17], NDM-1 was identified in K. pneumoniae and the $b l a_{\mathrm{NDM}-1}$ was applied in this study. It was cloned into TOPO- 100 expression vector and alanine mutations were generated as follows: NDM-2 (P28A), NDM-3 (D95A), NDM-4 (M154A), NDM-5 (V88A), NDM-7 (D130A), and NDM-9 (E152A). Changes in the activities of the alanine mutation variants were measured by E-test.

\section{Materials and Methods}

Detection of bla $a_{N D M-1}$ gene and cloning experiments

$b l a_{\mathrm{NDM}-1}$ gene was detected by PCR. Cloning of the $b l a_{\mathrm{NDM}-1}$ gene to expression vector $\mathrm{pET} 100 / \mathrm{D}-\mathrm{TOPO}+$ bla $_{\mathrm{NDM}-1}$ was amplified using primers Ndm_TOPO_Fw: 5'CACCATGGAATTGCCCAATATTATGC-3' and Ndm TOPO_Rw: 5'-TCAGCGCAGCTTGTCGGCCATGC-3' to obtain the whole gene sequence. The obtained PCR fragment was purified using a QIAquick column (QIAGEN, Courtaboeuf, France), cloned into the pET100/DTOPO vector, and transformed into $E$. coli $\mathrm{DH} 5 \alpha$ (Invitrogen Life Technologies, Saint Aubin, France). The transformant cells harboring plasmid vectors were selected on Mueller-Hinton (MH) agar containing ampicillin $(50 \mathrm{mg} / \mathrm{ml})$. The cloned DNA fragment inserted into one of the recombinant plasmids was sequenced by Macrogen. Sequencing results were analyzed using an alignment search tool BLAST (http://www.ncbi.nlm.nih.gov/BLAST) and the multiple sequence alignment program CLUSTALW2 (http://www.ebi.ac.uk/Tools/msa/ clustalw2/). 
Bioinformatics analysis

According to the official classification of $\beta$-lactamases web page, NDM-1 enzyme has 16 variants. The NDM-1 enzyme amino acid sequences were obtained from Genbank and amino acid changes were determined using the ClustalW program. Clinically important amino acids were identified and site-directed mutagenesis was performed on NDM-1 enzyme to substitute them. Clinically important amino acids were evaluated in this study.

\section{Site-directed mutation of the target amino acid}

All mutations were completed through $b l a_{\mathrm{NDM}-1}$ allele, which was cloned into the Champion ${ }^{\mathrm{TM}}$ pET100/D-TOPO expression vector. All alleles (NDM-2, NDM-3, NDM-4, NDM-5, NDM-7, and NDM-9) corresponding to the change in amino acids will be transformed into alanine amino acids by directed mutations out of the NDM-1 gene. Primers were designed to generate the alanine mutation in each allele (Table I). A single reaction mixture contained $2 \mu$ of plasmid DNA, $20 \mathrm{pM}$ of each primer, $10 \mu \mathrm{l}$ of reaction buffer, $3 \mu \mathrm{l}$ of $25 \mathrm{mM} \mathrm{MgCl}_{2}, 200 \mu \mathrm{M}$ of deoxynucleotide triphosphates, and 1.5 U of Pfu Polymerase (Promega, Madison, USA) in a final volume of $50 \mu \mathrm{l}$. All PCR results were analyzed on 1\% agarose containing $0.5 \mu \mathrm{g} / \mathrm{ml}$ ethidium bromide and were subsequently visualized under UV light. PCR products were cleaned up by PCR-clean up kit (Promega) and DpnI enzyme digestion was made. After digestion, the samples were transformed into $E$. coli DH5 $\alpha$. Plasmids were isolated and submitted to DNA sequence analysis.

Table I. The primers used to generate mutations

\begin{tabular}{ll}
\hline Target mutations & Primers $\left(5^{\prime} \rightarrow 3^{\prime}\right)$ \\
\hline NDM-2_28A_F & TTGATGCTGAGCGGGTGCATGGCCGGTGAAATCCGCCCGACGATT \\
NDM-2_28A_R & AATCGTCGGGCGGATTTCACCGGCCATGCACCCGCTCAGCATCAA \\
NDM-3_95A_F & GTGGTCGATACCGCCTGGACCGCTGACCAGACCGCCCAG \\
NDM-3_95A_R & CTGGGCGGTCTGGTCAGCGGTCCAGGCGGTATCGACCAC \\
NDM-4_154A_F & CAGCTTGCCCCGCAAGAGGGGGCGGTTGCGGCGCAACACAGC \\
NDM-4_154A_R & GCTGTGTTGCGCCGCAACCGCCCCCTCTTGCGGGGCAAGCTG \\
NDM-5_88A_F & AGGGATGGCGGCCGCGTGCTGGCGGTCGATACCGCCTGGACCGAT \\
NDM-5_88A_R & ATCGGTCCAGGCGGTATCGACCGCCAGCACGCGGCCGCCATCCCT \\
NDM-7_130A_F & CAGGACAAGATGGGCGGTATGGCCGCGCTGCATGCGGCGGGGATT \\
NDM-7_130A_R & AATCCCCGCCGCATGCAGCGCGGCCATACCGCCCATCTTGTCCTG \\
NDM-9_152A_F & TCGAACCAGCTTGCCCCGCAAGCGGGGATGGTTGCGGCGCAACACAGC \\
NDM-9_152A_F & GCTGTGTTGCGCCGCAACCATCCCCGCTTGCGGGGCAAGCTGGTTCGA \\
\hline
\end{tabular}

Note: NDM: New Delhi metallo- $\beta$-lactamase. 
Sequencing results were analyzed using BLAST (http://www.ncbi.nlm.nih.gov/ BLAST) and CLUSTALW2 (http://www.ebi.ac.uk/Tools/msa/clustalw2/).

E-test

The E-test was performed using E-test strips containing cefotaxim, piperacillin/tazobactam, cefepime, cefoxitin, piperacillin, ceftazidime, amoxicillin + clavulanic acid, imipenem, meropenem, and ertapenem, according to the manufacturer's instructions in plates with $\mathrm{MH}$ agar.

\section{Results}

NDM-type MBL enzyme has 16 variants and 14 of them reached the nucleotide sequence (Table II). Using specific primers, the $b l a_{\mathrm{NDM}-1}$ gene with 813 base pairs in length was amplified from $K$. pneumoniae genome and $b l a_{\mathrm{NDM}-1}$ gene was cloned to pET100/D-TOPO vector. Conversion of the targeted amino acids (P28A, D95A, M154A, V88A, D130A, and E152A) into alanine amino acid was confirmed by sequence analysis using pET100/D-TOPO + NDM vectors. The CCC codon encoding the $28^{\text {th }}$ amino acid (proline, P) was converted into the GCC codon encoding alanine (A) amino acid; the GAT codon encoding the $95^{\text {th }}$ amino acid (aspartic acid, D) was converted into the

Table II. NDM variants and amino acid changes that cause these variants

\begin{tabular}{lcccccccccccccc}
\hline Amino acids and positions, which cause alleles of NDM-1 & & & & & & \\
\hline NDM alleles & 28 & 32 & 36 & 69 & 74 & 88 & 95 & 130 & 152 & 154 & 200 & 222 & 233 \\
\hline NDM-1 & P & R & G & G & A & V & D & D & E & M & G & G & A \\
NDM-2 & A & R & G & G & A & V & D & D & E & M & G & G & A \\
NDM-3 & P & R & G & G & A & V & N & D & E & M & G & G & A \\
NDM-4 & P & R & G & G & A & V & D & D & E & $L$ & G & G & A \\
NDM-5 & P & R & G & G & A & L & D & D & E & $L$ & G & G & A \\
NDM-6 & P & R & G & G & A & V & D & D & E & M & G & G & $V$ \\
NDM-7 & P & R & G & G & A & V & D & $N$ & E & $L$ & G & G & A \\
NDM-8 & P & R & G & G & A & V & D & $G$ & E & $L$ & G & G & A \\
NDM-9 & P & R & G & G & A & V & D & D & $K$ & M & G & G & A \\
NDM-10 & P & S & D & S & $T$ & V & D & D & E & M & $R$ & G & A \\
NDM-11 & P & R & G & G & A & V & D & D & E & $V$ & G & G & A \\
NDM-12 & P & R & G & G & A & V & D & D & E & $L$ & G & $D$ & A \\
NDM-13 & P & R & G & G & A & V & $N$ & D & E & $L$ & G & G & A \\
NDM-14 & P & R & G & G & A & V & D & $G$ & E & M & G & G & A \\
\hline
\end{tabular}

Note: The elements in italics represent amino acid changes occurring in NDM-1 alleles. NDM: New Delhi metallo- $\beta$-lactamase. 
GCT codon encoding alanine (A) amino acid; the ATG codon encoding the $154^{\text {th }}$ amino acid (metyonin, M) was converted into the GCG codon encoding alanine (A) amino acid; the GTG codon encoding the $88^{\text {th }}$ amino acid (valine, V) was converted into the GCG codon encoding alanine (A) amino acid; the GAC codon encoding the $130^{\text {th }}$ amino acid (aspartic acid, D) was converted into the GCC codon encoding alanine (A) amino acid; and the GAG codon encoding the $152^{\text {th }}$ amino acid (glutamic acid, E) was converted into the GCC codon encoding alanine (A) amino acid.

Minimum inhibitory concentration (MIC) values were determined by E-test method. MIC values are shown in Table III. All mutant strains have $32 \mu \mathrm{g} / \mathrm{ml}$ MIC value for piperacillin/tazobactam except P28A mutant (MIC: $1.5 \mu \mathrm{g} / \mathrm{ml}$ ). While D130A and E152A have $256 \mu \mathrm{g} / \mathrm{ml}, \mathrm{P} 28 \mathrm{~A}$ and V88A have $0.125 \mu \mathrm{g} / \mathrm{ml}$ and D95A and M154A have $0.19 \mu \mathrm{g} / \mathrm{ml}$ MIC for cefepime. There are about 2,000-fold differences between the mutations of D130A and E152A and others (Table III). D95A and D130A have $2 \mu \mathrm{g} / \mathrm{ml}, \mathrm{P} 28 \mathrm{~A}$ and E152A have $4 \mu \mathrm{g} / \mathrm{ml}$, and there are twofold differences between them for cefoxitin. In addition, there is twofold difference between M154A $(1.5 \mu \mathrm{g} / \mathrm{ml})$ and V88A (3 $\mu \mathrm{g} / \mathrm{ml})$ for cefoxitin. P28A has the highest MIC ( $>2 \mu \mathrm{g} / \mathrm{ml})$ for ceftazidime. V88A and E152A have $48 \mu \mathrm{g} / \mathrm{ml}$, D95A and M154A have $24 \mu \mathrm{g} / \mathrm{ml}$, and P28A has $16 \mu \mathrm{g} / \mathrm{ml} \mathrm{MIC} \mathrm{for} \mathrm{amoxicillin} \mathrm{+}$ clavulanic acid. MIC of imipenem for D95A is $0.125 \mu \mathrm{g} / \mathrm{ml} ; 0.094 \mu \mathrm{g} / \mathrm{ml}$ for M154A, D130A, and E152A; and $0.032 \mu \mathrm{g} / \mathrm{ml}$ for P28A and V88A. There are twofold differences between P28A and D95A $(0.008 \mu \mathrm{g} / \mathrm{ml})$ and V88A, D130A, and $\mathrm{E} 152 \mathrm{~A}(0.016 \mu \mathrm{g} / \mathrm{ml})$.

\section{Discussion}

Creating the basic mechanisms of bacterial resistance to $\beta$-lactams is producing hydrolytic enzymes. These hydrolytic enzymes are called $\beta$-lactamases and they break the amide bond in the $\beta$-lactam ring of $\beta$-lactams $[18,19]$. To date, more than 1,000 $\beta$-lactamases were reported [20]. These enzymes are chromosomally encoded or on mobile genetic elements, such as transposons and plasmids. $\beta$-lactamases show too many differences according to their functional, biochemical, and similarity of amino acid sequences. According to Ambler molecular classification, $\beta$-lactamases can be structurally divided into two superfamilies: serin (classes A, C, and D) and MBLs (class B). Both serine $\beta$-lactamases and MBLs are able to hydrolyze the $\beta$-lactams, although catalytic mechanisms are different. The spread of MBLs among Gram-negative pathogens is a very serious problem [5]. More importantly, MBLs are carried with other resistance genes that restrict treatment options with the formation of multiresistance [6]. 


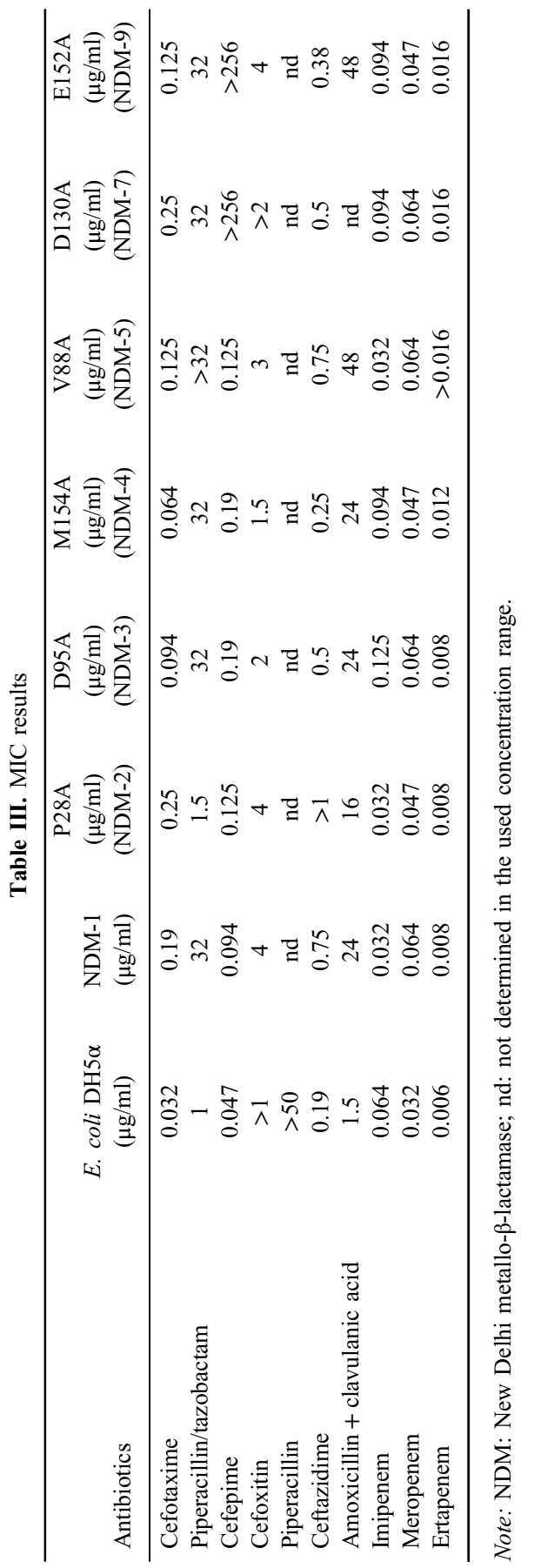


Rapid spread of MBL is related to mobile genetic elements, such as plasmids, transposable elements, and insertion sequences Cas9-like relationship between MBL genes [5, 6]. Spread of earned MBLs is very crucial for infection control and the treatment of patients [5]. NDM-type of metallo$\beta$-lactamases has been reported to have 16 variants and they have one or more amino acid changes between these variants (http://www.lahey.org/Studies/). Every year, some of the new variants are defined in Enterobacteriaceae. Accumulation of mutations on the gene takes years and the amount of the accumulated mutations on a gene that gives information about the gene has a long history. Currently, there are a few variants of the NDM-type $\beta$-lactamase when compared with other types of $\beta$-lactamases, but the number of the variants in future cannot be predicted or foreseen [21]. Site-directed mutagenesis experiments on MBL conducted on the results are of great importance, because occurring variants of increasing antibiotic concentrations (MICs) contain mutations selected on their ability to remain alive in the bacterial host. Therefore, experiments with purified recombinant proteins increase the understanding about the effects of mutations that confer resistance [21].

Yildiz et al. [22] have conducted phenotypic tests on carbapenemaseproducing strains (OXA-48 and NDM-1) and they found that CarbaNP and carbapenem inactivation method tests were suitable for describing carbapenem producers. In a study, NDM-5 has been shown to increase the MIC values for ceftazidime, cefotaxime, and cefepime comparing with NDM-1 [23]. In another study on NDM variant of antibiotic susceptibility profile, an increase in MIC values against carbapenems has been observed in NDM-5 and NDM-7 [24]. Another work showed that the NDM-producing isolates exhibited high meropenem MIC values of $2-4 \mathrm{~g} / \mathrm{ml}$ [25].

In this study, substitution of valine at the $88^{\text {th }}$ position to alanine, when compared with NDM-1, appears to give almost similar MIC values. In this work, NDM enzyme substrate profile differs among NDMs by means of in vitro assays directed mutation MBL-out made alanine mutation resulting mutant. The rapid evolution of NDM gene and intercontinental travel and health tourism role illustrate the global spread of multidrug-resistant organisms. Because of the rapid spread of NDM gene, it is expected that more will be discovered in future.

\section{Conflict of Interest}

The author declares no conflict of interest. 


\section{References}

1. Yong, D., Toleman, M. A., Giske, C. G., Cho, H. S., Sundman, K., Lee, K., Walsh, T. R.: Characterization of a new metallo- $\beta$-lactamase gene, blaNDM-1, and a novel erythromycin esterase gene carried on a unique genetic structure in Klebsiella pneumoniae sequence type 14 from India. Antimicrob Agents Chemother 53, 5046-5054 (2009).

2. Nordmann, P., Poirel, L., Walsh, T. R., Livermore, D. M.: The emerging NDM carbapenemases. Trends Microbiol 19, 588-595 (2011).

3. Johnson, A. P., Woodford, N.: Global spread of antibiotic resistance: The example of New Delhi metallo $\beta$-lactamase (NDM)-mediated carbapenem resistance. J Med Microbiol 62, 499-513 (2013).

4. Kaase, M., Nordmann, P., Wichelhaus, T. A., Gatermann, S. G., Bonnin, R. A., Poirel, L.: NDM-2 carbapenemase in Acinetobacter baumannii from Egypt. J Antimicrob Chemother 66, 1260-1262 (2011).

5. Cornaglia, G., Giamarellou, H., Rossolini, G. M.: Metallo $\beta$-lactamase: A last frontier for $\beta$-lactams? Lancet Infect Dis 11, 381-393 (2011).

6. Walsh, T. R., Toleman, M. A., Poirel, L., Nordmann, P.: Metallo- $\beta$-lactamases: The quiet before the storm? Clin Microbiol Rev 18, 306-325 (2005).

7. Queenan, A. M., Bush, K.: Carbapenemases: The versatile $\beta$-lactamases. Clin Microbiol Rev 20, 440-458 (2007).

8. Kumarasamy, K. K., Toleman, M. A., Walsh, T. R., Bagaria, J., Butt, F., Balakrishnan, R., Chaudhary, U., Doumith, M., Giske, C. G., Irfan, S., Krishnan, P., Kumar, A. V., Maharjan, S., Mushtaq, S., Noorie, T., Paterson, D. L., Pearson, A., Perry, C., Pike, R., Rao, B., Ray, U., Sarma, J. B., Sharma, M., Sheridan, E., Thirunarayan, M. A., Turton, J., Upadhyay, S., Warner, M., Welfare, W., Livermore, D. M., Woodford, N.: Emergence of a new antibiotic resistance mechanism in India, Pakistan, and the UK: A molecular, biological, and epidemiological study. Lancet Infect Dis 10, 597-602 (2010).

9. Castanheira, M., Deshpande, L. M., Mathai, D., Bell, J. M., Jones, R. N., Mendes, R. E.: Early dissemination of NDM-1 and OXA-181-producing Enterobacteriaceae in Indian hospitals: Report from the SENTRY Antimicrobial Surveillance Program, 2006-2007. Antimicrob Agents Chemother 55, 1274-1278 (2011).

10. Krishna, B. V.: New Delhi metallo-beta-lactamases: A wake-up call for microbiologists. Indian J Med Microbiol 28, 265-266 (2010).

11. Mochon, A. B., Garner, O. B., Hindler, J. A., Krogstad, P., Ward, K. W., Lewinski, M. A., Rasheed, J. K., Anderson, K. F., Limbago, B. M., Humphries, R. M.: New Delhi metallo- $\beta-$ lactamase (NDM-1) producing Klebsiella pneumoniae: Case report and laboratory detection strategies. J Clin Microbiol 49, 1667-1670 (2011).

12. Muir, A., Weinbren, M. J.: New Delhi metallo-beta-lactamase: A cautionary tale. J Hosp Infect 75, 236-246 (2010).

13. Karthikeyan, K., Thirunarayan, M. A., Krishnan, P.: Coexistence of blaOXA-23 with blaNDM-1 and armA in clinical isolates of Acinetobacter baumannii from India. $\mathbf{J}$ Antimicrob Chemother 65, 2253-2254 (2010).

14. Chen, Z., Qlu, S., Wang, Y., Wang, Y., Liu, S., Wang, Z., Du, X., Wang, L., Guo, J., Wang, Z., Liu, N., Yuan, J., Song, H., Huang, L.: Coexistence of blaNDM-1 with the prevalent blaOXA-23 and blaIMP in pan-drug resistant Acinetobacter baumannii isolates in China. Clin Infect Dis 52, 692-693 (2011). 
15. Rimrang, B., Chanawong, A., Lulitanond, A., Wilailuckana, C., Charoensri, N., Sribenjalux, P., Phumsrikaew, W., Wonglakorn, L., Kerdsin, A., Chetchotisakd, P.: Emergence of NDM-1- and IMP-14a-producing Enterobacteriaceae in Thailand. J Antimicrob Chemother 67, 2626-2630 (2012).

16. Poirel, L., Ozdamar, M., Ocampo-Sosa, A. A., Türkoglu, S., Ozer, U. G., Nordmann, P.: NDM-1-producing Klebsiella pneumoniae now in Turkey. Antimicrob Agents Chemother 56, 2784-2785 (2012).

17. Iraz, M., Düzgün, A. Ö., Sandallı, C., Doymaz, M. Z., Akkoyunlu, Y., Saral, A., Peleg, A. Y., Özgümüş, O. B., Beriş, F. Ş., Karaoğlu, H., Çiçek, A. Ç.: Distribution of $\beta$-lactamase genes among carbapenem-resistant Klebsiella pneumoniae strains isolated from patients in Turkey. Ann Lab Med 6, 595-601 (2015).

18. Essack, S. Y.: The development of $\beta$-lactam antibiotics in response to the evolution of $\beta$-lactamases. Pharm Res 18, 1391-1399 (2001).

19. Siu, L. K.: Antibiotics: Action and resistance in Gram-negative bacteria. J Microbiol Immunol Infect 35, 1-11 (2002).

20. Davies, J., Davies, D.: Origins and evolution of antibiotic resistance. Microbiol Mol Biol Rev 74, 417-433 (2010).

21. Meini, M. R., Llarrull, L. I., Vila, A. J.: Evolution of metallo- $\beta$-lactamases: Trends revealed by natural diversity and in vitro evolution. Antibiotics (Basel) 3, 285-316 (2014).

22. Yıldız, S. S., Kaşkatepe, B., Avcıküçük, H., Öztürk, Ş.: Performance of CarbaNP and CIM tests in OXA-48 carbapenemase-producing Enterobacteriaceae. Acta Microbiol Immunol Hung 64, 9-16 (2017).

23. Hornsey, M., Phee, L., Wareham, D. W.: A novel variant, NDM-5, of the New Delhi metallo- $\beta$-lactamase in a multidrug-resistant Escherichia coli ST648 isolate recovered from a patient in the United Kingdom. Antimicrob Agents Chemother 55, 5952-5954 (2011).

24. Makena, A., Brem, J., Pfeffer, I., Geffen, R. E., Wilkins, S. E., Tarhonskaya, H., Flashman, E., Phee, L. M., Wareham, D. W., Schofield, C. J.: Biochemical characterization of New Delhi metallo- $\beta$-lactamase variants reveals differences in protein stability. J Antimicrob Chemother 70, 463-469 (2015).

25. Fattouh, R., Tijet, N., McGeer, A., Poutanen, S. M., Melano, R. G., Patel, S. N.: What is the appropriate meropenem MIC for screening of carbapenemase-producing Enterobacteriaceae in low-prevalence settings? Antimicrob Agents Chemother 60, 1556-1559 (2015). 